\title{
Article \\ Computational Methods to Study Human Transcript Variants in COVID-19 Infected Lung Cancer Cells
}

\author{
Jiao Sun ${ }^{1,2,+}$, Naima Ahmed Fahmi ${ }^{1,2,+}$, Heba Nassereddeen ${ }^{2,3}$, Sze Cheng ${ }^{4} \mathbb{D}$, Irene Martinez ${ }^{5}$, Deliang Fan ${ }^{6}$, \\ Jeongsik Yong $4, *(\mathbb{D})$ and Wei Zhang $1,2, *$ (D)
}

1 Department of Computer Science, University of Central Florida, Orlando, FL 32816, USA; jiao.sun@knights.ucf.edu (J.S.); fnaima@knights.ucf.edu (N.A.F.)

2 Genomics and Bioinformatics Cluster, University of Central Florida, Orlando, FL 32816, USA; hebanasser@knights.ucf.edu

3 Department of Electrical and Computer Engineering, University of Central Florida, Orlando, FL 32816, USA

4 Department of Biochemistry, Molecular Biology and Biophysics, University of Minnesota Twin Cities, Minneapolis, MN 55455, USA; cheng705@umn.edu

5 Department of Molecular Biotechnology, Universität Heidelberg, 69120 Heidelberg, Germany; irene.martinez@stud.uni-heidelberg.de

6 School of Electrical, Computer and Energy Engineering, Arizona State University, Tempe, AZ 85287, USA ; dfan@asu.edu

* Correspondence: jyong@umn.edu (J.Y.); wzhang.cs@ucf.edu (W.Z.)

+ These authors have contributed equally to this work and share first authorship.

Citation: Sun, J.; Fahmi, N.A.; Nassereddeen, H.; Cheng, S.; Martinez, I.; Fan, D.; Yong, J.; Zhang, W. Computational Methods to Study Human Transcript Variants in COVID-19 Infected Lung Cancer Cells. Int. J. Mol. Sci. 2021, 22, 9684. https://doi.org/10.3390/ijms22189684

Academic Editor: Daisuke Kaida

Received: 1 July 2021

Accepted: 30 August 2021

Published: 7 September 2021

Publisher's Note: MDPI stays neutral with regard to jurisdictional claims in published maps and institutional affiliations.

Copyright: (c) 2021 by the authors. Licensee MDPI, Basel, Switzerland. This article is an open access article distributed under the terms and conditions of the Creative Commons Attribution (CC BY) license (https:// creativecommons.org/licenses/by/ $4.0 /)$.

\begin{abstract}
Microbes and viruses are known to alter host transcriptomes by means of infection. In light of recent challenges posed by the COVID-19 pandemic, a deeper understanding of the disease at the transcriptome level is needed. However, research about transcriptome reprogramming by post-transcriptional regulation is very limited. In this study, computational methods developed by our lab were applied to RNA-seq data to detect transcript variants (i.e., alternative splicing (AS) and alternative polyadenylation (APA) events). The RNA-seq data were obtained from a publicly available source, and they consist of mock-treated and SARS-CoV-2 infected (COVID-19) lung alveolar (A549) cells. Data analysis results show that more AS events are found in SARS-CoV-2 infected cells than in mock-treated cells, whereas fewer APA events are detected in SARS-CoV-2 infected cells. A combination of conventional differential gene expression analysis and transcript variants analysis revealed that most of the genes with transcript variants are not differentially expressed. This indicates that no strong correlation exists between differential gene expression and the AS/APA events in the mock-treated or SARS-CoV-2 infected samples. These genes with transcript variants can be applied as another layer of molecular signatures for COVID-19 studies. In addition, the transcript variants are enriched in important biological pathways that were not detected in the studies that only focused on differential gene expression analysis. Therefore, the pathways may lead to new molecular mechanisms of SARS-CoV-2 pathogenesis.
\end{abstract}

Keywords: COVID-19; transcript variants; alternative splicing; alternative polyadenylation; RNAseq; $3^{\prime}$-UTR

\section{Introduction}

Recent research on the severe acute respiratory syndrome coronavirus 2 (SARS-CoV-2) has focused primarily on gene expression profiling in various human cell lines and patient samples in an effort to understand the molecular basis of SARS-CoV-2 pathogenesis [1-3]. Unsurprisingly, many of the differentially expressed host genes identified are involved in the antiviral response $[1,4]$. As the SARS-CoV-2 virus can trigger different levels of changes in humans, a thorough understanding of molecular signatures beyond gene expression profiling is needed to comprehensively dissect the pathogenesis of SARS-CoV-2 coronavirus. 
Immune activation to viral infection is regulated by changes in gene expression and post-transcriptional regulation, such as AS and APA [5,6]. A classic example of AS in immune cells is the generation of neoantigens, novel peptides of immune proteins for antibody diversification in normal conditions [7]. However, during viral infection by the influenza A virus, the virus can induce widespread AS in the host genes $[5,7,8]$. Mechanically, viral proteins can interact with splicing and polyadenylation factors and cause misprocessing of the host mRNAs [8]. Some of these isoforms may encode unique neoantigens or disrupt critical functional domains, thereby promoting viral replication and production [7-9]. These studies stress the importance of the RNA-processing regulation upon viral infection. A recent study suggests that the NSP16 viral protein of SARS-CoV-2 can interact with the human U1 and U2 snRNA, thereby changing the AS landscape in humans [10]. This warranted our investigation of the host splicing alteration during SARS-CoV-2 infection to further reveal the molecular basis of the COVID-19 disease.

Here, RNA-seq data of SARS-CoV-2 infected lung cancer (A549) cells are used to investigate the SARS-CoV-2-inducted remodeling of the host transcriptome [1]. By utilizing computational methods and pipelines, including custom-developed tools, we have identified global AS and APA (Figure 1) changes upon SARS-CoV-2 infection in human lung epithelial cells. These widespread splicing signatures rarely overlap with findings from differential gene expression studies and are enriched in many previously undetected pathways critical for SARS-CoV-2 pathobiology.

A

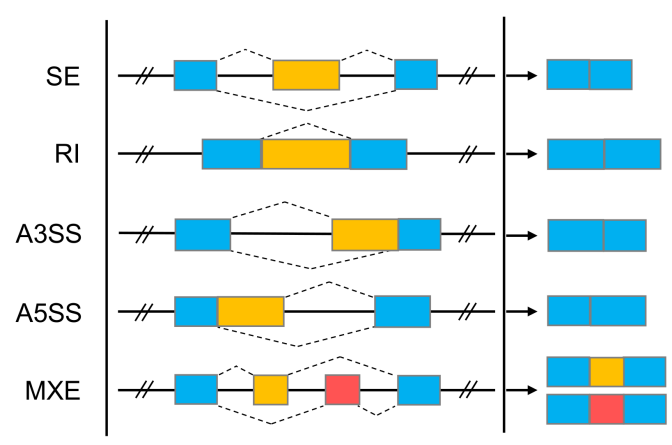

B

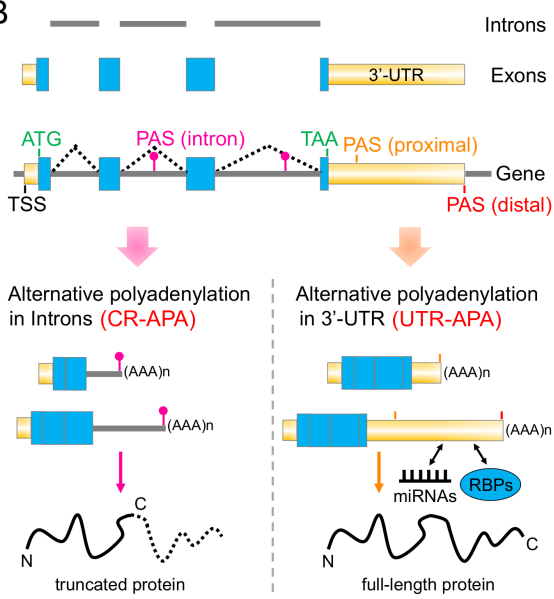

Figure 1. Alternative splicing (AS) and alternative polyadenylation (APA). (A) Schematic representation of the five major types of alternative splicing in eukaryotes: Skipped Exon (SE), Retained Intron (RI), Alternative $3^{\prime}$ Splice Site (A3SS), Alternative 5' Splice Site (A5SS), and Mutually Exclusive Exon (MXE). Light blue boxes represent the constitutive exons, whereas light yellow and pink exons represent the spliced ones. (B) Two types of alternative polyadenylation, i.e., coding region alternative polyadenylation (CR-APA) and 3'-UTR alternative polyadenylation (UTR-APA), and their impact on the functional proteome. PAS: polyadenylation signal; TSS: transcription start site; RBP: RNA-binding protein.

\section{Results}

In this study, multiple bioinformatics pipelines and statistical methods were used to identify the molecular signatures of COVID-19 at the transcriptional and post-transcriptional levels. The objective is to achieve a better understanding of the impact of transcriptomic changes on the proteome in COVID-19. In this section, we first describe previously published RNA-seq data of COVID-19 [1] that are used in this study (Section 2.1). Then in the following subsections, we focus on reporting the results of our analyses on transcriptome changes using these datasets: we will extensively discuss the alterations of splicing and polyadenylation events as well as the differential expression of genes/transcripts upon SARS-CoV-2 infection in A549 human lung cancer cells. 


\subsection{Data}

The RNA-seq data used in this study were downloaded from the NCBI Gene Expression Omnibus (GEO) database, under the accession number GSE147507 [1]. The dataset contains independent biological triplicates that were mock-treated or infected with SARS-CoV-2. Series 2, 5, and 7 in the dataset were analyzed in this study. Series 2 and 5 consist of transformed lung alveolar (A549) samples that are mock-treated or infected with SARS-CoV-2. Series 7 includes samples from transformed lung-derived Calu-3 cells that are mock-treated or infected with SARS-CoV-2.

We used TopHat2 [11] to conduct sequence alignment. These three pairs of cell line data (Series 2, 5, and 7) with a high overall alignment rate were selected for downstream analyses (Supplementary Table S1). Series 5 results are discussed in this manuscript, whereas results for the other two series can be found in the Supplementary Materials (Supplementary Tables S2-S8). The RNA-seq data were quantified with Kallisto [12] for differential transcript and gene expression analyses. To generate the read coverage profile, SAMtools [13] was used with the aligned bam files as an input.

\subsection{Alternative Splicing}

Five major AS events between groups of mock-treated and SARS-CoV-2 infected A549 cells are reported in Table 1 . The table displays information regarding the number of AS events in each category. Overall, more AS events were detected in SARS-CoV-2 infected cells than in mock-treated ones. Among the five types of AS events, the Skipped Exon (SE) category was found to be the most common type; 51 AS events were identified in the mock-treated cells, and 126 AS events were identified in the SARS-CoV-2 infected cells. Notably, compared to other types of AS events, the AS event for alternative 5' splice site (A5SS) was more frequent in the SARS-CoV-2 infected cells. We further investigated the top 10 significant events for the AS type SE among the two groups (Table 2). The AS events are sorted in ascending order by the $p$-value, as defined in the Chi-squared hypothesis test. The column 'Ratio Difference' denotes the differences in the ratio of average read coverage between the SARS-CoV-2 infected and mock-treated cells. A positive value for the ratio difference indicates that the mock-treated cells have more SE splicing events compared to the SARS-CoV-2 infected cells, and a negative value means the other way around.

Table 1. Number of detected significant splicing events in each category between SARS-CoV-2 infected and mock-treated cells.

\begin{tabular}{cccccc}
\hline Sample & SE & RI & MXE & A3SS & A5SS \\
\hline Mock & 51 & 16 & 7 & 13 & 6 \\
SARS-CoV-2 & 126 & 30 & 19 & 59 & 100 \\
\hline
\end{tabular}

Table 2. Top 10 significant splicing events between A549 mock-treated and SARS-CoV-2 infected cells.

\begin{tabular}{ccccccc}
\hline Gene Name & Chr & Start & End & $p$-Value & FDR & Ratio Difference \\
\hline TPT1 & chr13 & $45,914,846$ & $45,914,920$ & $5.81 \times 10^{-18}$ & $7.5 \times 10^{-14}$ & -0.074 \\
C6ORF48 & chr6 & $3,118,955$ & $3,119,049$ & $9.21 \times 10^{-15}$ & $5.94 \times 10^{-11}$ & -0.113 \\
FKBP1A & chr20 & $1,373,477$ & $1,373,525$ & $2.25 \times 10^{-14}$ & $9.67 \times 10^{-11}$ & -0.168 \\
PPIA & chr7 & $44,838,346$ & $44,838,413$ & $1.63 \times 10^{-12}$ & $5.25 \times 10^{-9}$ & 0.149 \\
HNRNPA1 & chr12 & $54,676,862$ & $54,677,018$ & $1.26 \times 10^{-11}$ & $3.26 \times 10^{-8}$ & -0.248 \\
RPS24 & chr10 & $79,799,961$ & $79,799,983$ & $5.25 \times 10^{-11}$ & $1.13 \times 10^{-7}$ & 0.122 \\
RPS9 & chr19 & $54,710,420$ & $54,710,592$ & $4.59 \times 10^{-10}$ & $8.46 \times 10^{-7}$ & 0.117 \\
SRSF2 & chr17 & $74,731,853$ & $74,731,957$ & $1.24 \times 10^{-7}$ & $1.78 \times 10^{-4}$ & 0.172 \\
CA12 & chr15 & $63,638,728$ & $63,638,908$ & $1.66 \times 10^{-7}$ & $1.99 \times 10^{-4}$ & -0.103 \\
RPLP1 & chr15 & $69,745,985$ & $69,746,060$ & $1.69 \times 10^{-7}$ & $1.99 \times 10^{-4}$ & 0.040 \\
\hline
\end{tabular}

From the differential AS analysis performed in Table 1, we conducted a literature survey of the genes with significant AS events. Three of them are implicated in SARS-CoV-2 
pathogenesis and prognosis as shown in Table 3. In addition, the genes with the splicing events were analyzed with the DAVID functional annotation tool [14]. The enriched KEGG pathways in mock-treated and SARS-CoV-2 infected cells are shown in Figure 2A. Interestingly, genes with AS events are enriched in ribosome and spliceosome, suggesting that SARS-CoV-2 infected cells undergo changes in their gene expression pathways.

Table 3. Literature review of identified genes with transcript variants and their implication on SARS-CoV-2 pathogenesis and prognosis.

\begin{tabular}{|c|c|c|c|}
\hline Category & Gene & Ref. & Description \\
\hline \multirow{3}{*}{$\begin{array}{l}\text { Alternative } \\
\text { Splicing }\end{array}$} & BTF3 & [15] & $\begin{array}{l}\text { Interacts with the NSP10 CoV protein, which is involved in the pathological function of } \\
\text { SARS-CoV in cells. }\end{array}$ \\
\hline & FKBP1A & [16] & FKBP1A causes immunosuppression and is required by CoV for viral growth. \\
\hline & G3BP1 & [17] & $\begin{array}{l}\text { SARS-CoV-2 N protein undergoes liquid-liquid phase separation, which serves as a } \\
\text { scaffold for virus replication and assembly, through its N-terminal intrinsically } \\
\text { disordered region (IDR) with G3BP1. }\end{array}$ \\
\hline \multirow[t]{3}{*}{ UTR-APA } & ANXA2 & [18] & $\begin{array}{l}\text { The upregulation of expression of annexin A2 (ANXA2) by SARS-associated cytokines and } \\
\text { the cross-reactivity of anti-SARS-CoV S2 antibodies to annexin A2 may have implications } \\
\text { in SARS disease pathogenesis. }\end{array}$ \\
\hline & CAV1 & [19] & Coronaviruses enter cells via the CAV1 dependent pathway. \\
\hline & TMEM97 & [20] & TMEM97 forms a complex with ACE2 and modulates its ability to internalize the SARS-CoV-2. \\
\hline \multirow{3}{*}{ CR-APA } & CTSC & [21] & $\begin{array}{l}\text { CTSC activates the elastase-related neutrophil proteases mediated tissue degradation } \\
\text { in which it diffuses the alveolar inflammation in acute respiratory distress syndrome. }\end{array}$ \\
\hline & RHOA & [22] & $\begin{array}{l}\text { Activation of RhoA GTPase and its downstream effector, Rho kinase (ROCK), contributes to } \\
\text { a burst in inflammatory features, immune cell migration, apoptosis, coagulation, contraction, } \\
\text { and cell adhesion in pulmonary endothelial cells, leading to endothelium barrier dysfunction } \\
\text { and edema as hallmarks of lung injury. }\end{array}$ \\
\hline & CANX & [23] & Calnexin (CANX) strictly monitors the maturation of the CoV S protein by its direct binding. \\
\hline \multirow{2}{*}{$\begin{array}{l}\text { Differential } \\
\text { Expression }\end{array}$} & BCL2A1 & [24] & $\begin{array}{l}\text { Pro-survival gene mostly present in adult genes, if downregulated, promotes apoptosis in } \\
\text { lung tissue. }\end{array}$ \\
\hline & SKP2 & [25] & $\begin{array}{l}\text { SKP2 attenuates autophagy through Beclin1-ubiquitination and allowing for the replication } \\
\text { of coronaviruses. }\end{array}$ \\
\hline
\end{tabular}

Figure 2B shows a high resolution read coverage plot generated by our AS-Quant pipeline [26] for the gene SDCCAG3 with a significant AS event. The figure illustrates the notable alternation of expression levels in the spliced exons between the mock-treated and SARS-Cov-2 infected groups.

\subsection{Alternative Polyadenylation}

To further assess the predictions of alternative polyadenylation events between mocktreated and the SARS-CoV-2 infected cells, both CR-APA and UTR-APA events (Figure 1B) were identified by the computational pipelines introduced in Section 4.2.

\subsubsection{UTR-APA}

Our pipeline, APA-Scan [27], identified 144 unannotated UTR-APA events, which were reported using the Chi-squared hypothesis test on A549 cells. A total of 137 UTRAPA events were identified in mock-treated cells, while only 7 UTR-APA events were found in SARS-CoV-2 infected cells, indicating that UTR-APA events were suppressed in SARS-CoV-2 infected cells. Figure 3 shows a UTR-APA event for the gene HNRNPH3 generated by APA-Scan. In Table 4, the top 10 UTR-APA events are reported by the $p$ value in the ascending order. The $p$-values indicate the significance of the UTR-APA event between mock versus SARS-CoV-2 infected cells. The ratio difference in the last column is calculated by taking the average read coverage ratios of the two groups. A positive ratio difference indicates an increase in the UTR-APA event in mock-treated cells. Based on the results reported in Table 4, all the top 10 events were identified from the mock-treated cells, thereby reinforcing that SARS-CoV-2 infections suppress the usage of the proximal 
polyadenylation signal in the $3^{\prime}$-end processing of mRNAs. The literature survey shows that three genes (ANXA2, CAV1, and TMEM97) with the UTR-APA events related to SARSCoV-2 pathogenesis and prognosis (Table 3). For example, TMEM97 has been recently shown to interact with SARS-CoV-2 viral proteins [20].

A

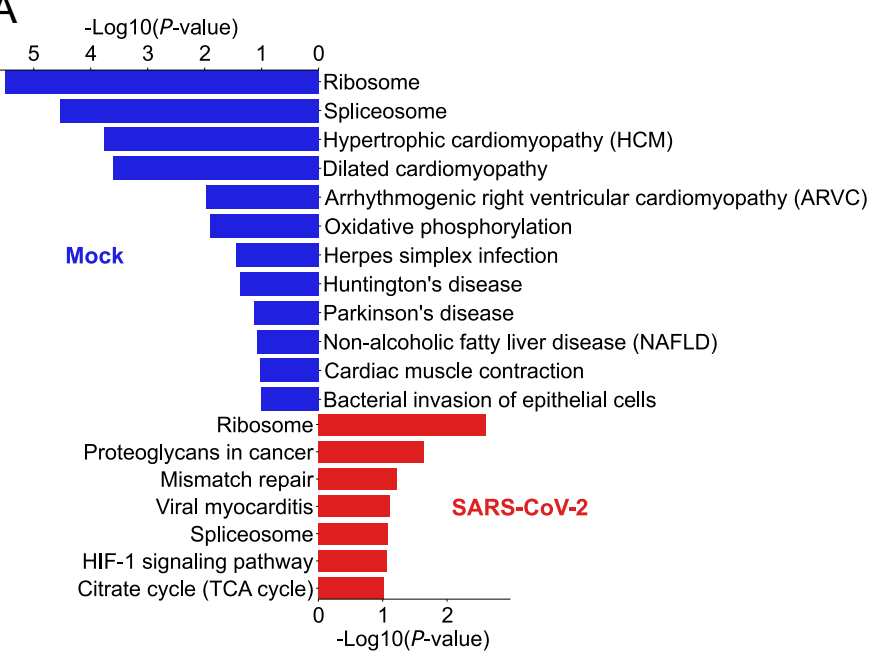

B

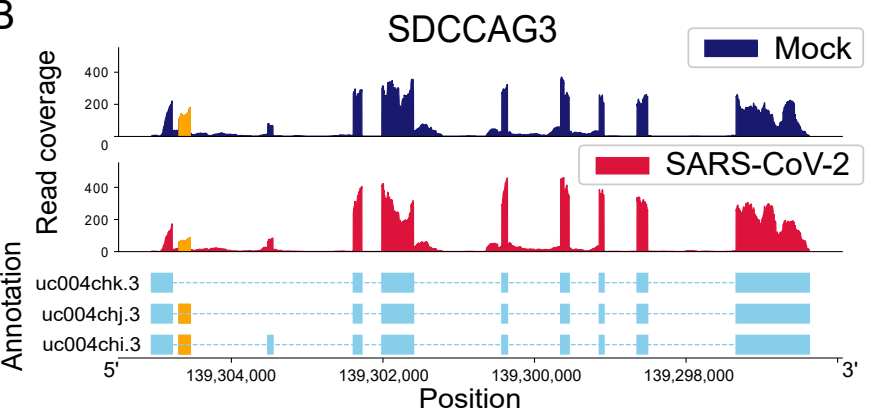

Figure 2. (A) KEGG pathways enriched by alternative splicing events. The blue and red bar charts show the pathways enriched by the splicing events in mock-treated cells and SARS-CoV-2 infected cells, respectively. (B) A plot for the SDCCAG3 AS event. The spliced exon is highlighted in orange. The first two subplots show the read coverage of the gene in both groups, whereas the bottom subplot denotes the gene annotation with exon information. The $x$-axis and $y$-axis of the plot represent the position of the specific gene and read coverage of that sample, respectively. To show the differential splicing event, the altered exon is highlighted in orange to provide a clear insight into the phenomenon.

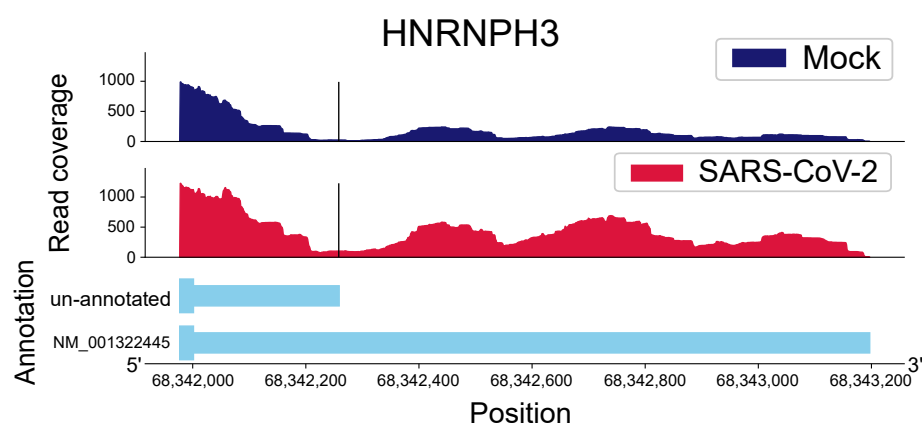

Figure 3. One example of a gene (HNRNPH3) shows an alternative polyadenylation event in the $3^{\prime}$-UTR region. The black vertical line indicates the potential cleavage site. The $x$-axis and $y$-axis represent the position of the gene in its chromosome and the read coverage, respectively. The top two subplots show the changes in read coverage surrounding the event, and the cleavage site is indicated by a black vertical line. The bottom part of the plot shows both the full length and truncated $3^{\prime}$-UTRs. 
Table 4. Top 10 significant alternative polyadenylation events in the $3^{\prime}$-UTR region (UTR-APA) between A549 mock-treated and SARS-CoV-2 infected cells. The 'Position' column refers to the potential cleavage site for the UTR-APA.

\begin{tabular}{cccccc}
\hline Gene Name & Chr & Position & $p$-Value & FDR & Ratio Difference \\
\hline ACTN4 & chr19 & $38,730,184$ & $6.07 \times 10^{-14}$ & $5.71 \times 10^{-10}$ & 0.144 \\
ALDH1A1 & chr9 & $72,900,986$ & $1.34 \times 10^{-12}$ & $6.30 \times 10^{-9}$ & 0.054 \\
S100A6 & chr1 & $153,534,690$ & $2.73 \times 10^{-11}$ & $8.56 \times 10^{-8}$ & 0.045 \\
HNRNPA2B1 & chr7 & $26,191,861$ & $1.26 \times 10^{-9}$ & $2.96 \times 10^{-6}$ & 0.088 \\
PMEPA1 & chr20 & $57,651,579$ & $2.10 \times 10^{-9}$ & $3.95 \times 10^{-6}$ & 0.153 \\
ACAT2 & chr6 & $159,779,033$ & $3.11 \times 10^{-9}$ & $4.88 \times 10^{-6}$ & 0.273 \\
ARL4C & chr2 & $234,495,151$ & $6.05 \times 10^{-8}$ & $8.14 \times 10^{-5}$ & 0.231 \\
H3F3A & chr1 & $226,071,775$ & $8.70 \times 10^{-8}$ & $1.02 \times 10^{-4}$ & 0.189 \\
RPL15 & chr3 & $23,919,537$ & $1.32 \times 10^{-7}$ & $1.38 \times 10^{-4}$ & 0.041 \\
MYH9 & chr22 & $36,281,660$ & $3.29 \times 10^{-7}$ & $2.91 \times 10^{-4}$ & 0.177 \\
\hline
\end{tabular}

\subsubsection{CR-APA}

In the CR-APA analysis, both the hg38 RefSeq annotation [28] and the UCSC annotation [29] were applied to report the list of CR-APA events between mock-treated versus SARS-CoV-2 infected cells. We identified significant CR-APA events by measuring the CR-truncation ratio (Equation (4)). We found an increase of $1206 \mathrm{CR}$-APA events in mock-treated cells and an enrichment of 656 CR-APA events in SARS-CoV-2 infected cells, indicating that CR-APA events are more frequent in mock-treated cells. The bipartite expression patterns of CR-APA in Figure 4 revealed an enrichment of specific truncated mRNAs in both mock and SARS-CoV-2 infected cells, indicating that reprogramming of the transcriptome and consequent changes in the proteome diversity would occur upon SARS-CoV-2 infection.

Among the significant CR-APA events, the top 10 significant events are listed in Table 5. The ratio difference is calculated by taking the difference between the average CR-truncation ratio of the SARS-CoV-2 infected cells and mock-treated cells. A CR-APA event occurs in SARS-CoV-2 infected samples if the ratio difference is larger than zero. It is considered significant in the mock-treated group if the ratio difference is smaller than zero. A literature review shows that several genes with CR-APA events are connected to SARS-CoV-2 pathogenesis and prognosis, as described in Table 3. The KEGG pathways in mock-treated and the SARS-Cov-2 infected cells are shown in Figure 5A. Particularly, genes involved in oxidative phosphorylation increased CR-APA, which may affect the expression of full-length proteins that are critical for cellular energy production and mitochondrial dysfunction in respiratory illnesses [30]. It was shown that one of the SARS-CoV-2 proteins, NSP16, binds to the mRNA recognition domains of the spliceosome and suppresses global mRNA splicing in SARS-CoV-2-infected human cells [10]. The KEGG pathways enriched in Figures 2A and 5A show AS and CR-APA events in genes associated with the spliceosome pathway, suggesting that alterations in the regulatory splicing pathway could happen. This, in turn, would affect splicing and splicing-coupled CR-APA events upon SARS-CoV-2 infection, leading to further deleterious effects on gene function [31].

Figure 5B shows a CR-APA event for the gene LARP6. The top two subplots show the read coverage of the gene in two conditions. The bottom panel represents the isoform annotations. NM_197958 is an annotated CR-truncated isoform in the gene, and the last highlighted exon indicates the end of the CR-APA transcript. Compared to SARSCoV-2 infected cells, the plot shows a significant increase of RNA-seq alignments in the highlighted exon, indicating a higher CR-truncation ratio of LARP6 in mock-treated cells compared to SARS-CoV-2 infected cells. 


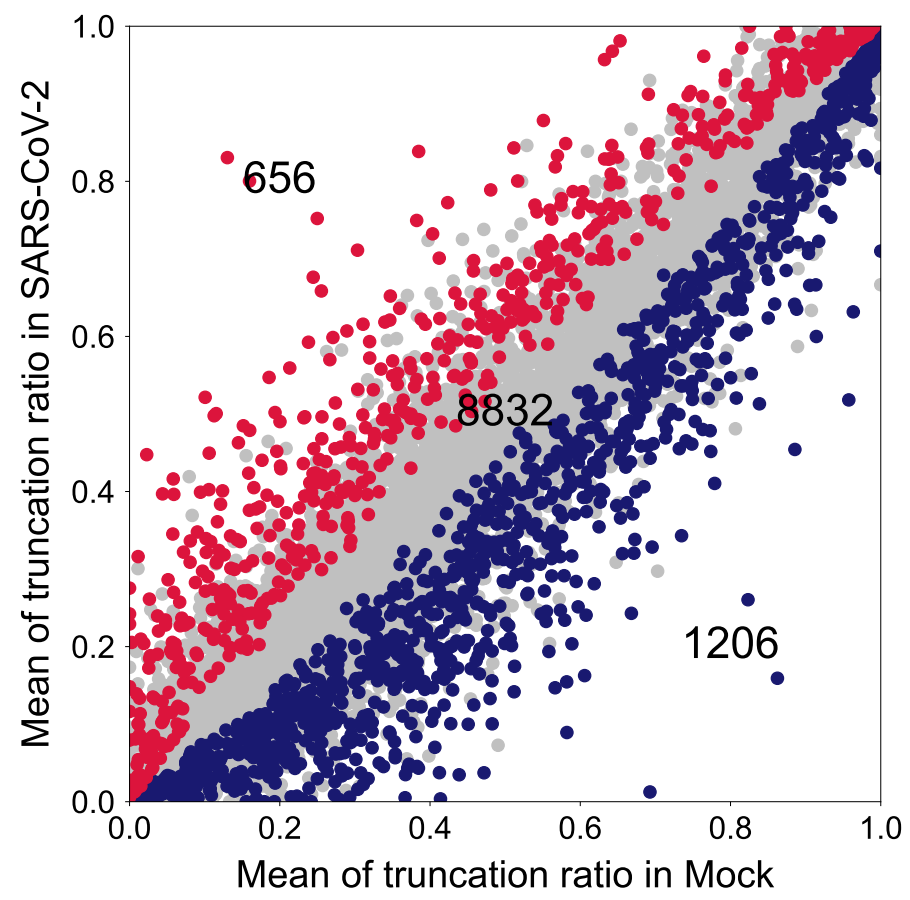

Figure 4. RNA-seq data of mock-treated and SARS-CoV-2 infected A549 cells were analyzed for the CR-APA. The $x$-axis and $y$-axis represent the CR-truncation ratios ((short mRNA)/(total mRNA)) of a gene. Each dot represents a gene, where the blue dots and red dots show the significant ones in mock and SARS-CoV-2 infected cells, respectively. Upon SARS-CoV-2 infection, 656 genes showed up-regulated CR-APA while 1206 genes showed down-regulated CR-APA. A total of 8832 genes remained unchanged in their CR-APA.

Table 5. Top 10 significant alternative polyadenylation events in the coding region (CR-APA) between A549 mock-treated and SARS-CoV-2 infected cells.

\begin{tabular}{cccccc}
\hline Gene & Chr & Truncated Position & $p$-Value & FDR & Ratio Difference \\
\hline GLRX5 & chr14 & $95,544,557$ & $2.76 \times 10^{-6}$ & $6.12 \times 10^{-3}$ & 0.361 \\
UBXN6 & chr19 & $4,445,009$ & $5.98 \times 10^{-6}$ & $1.14 \times 10^{-2}$ & 0.020 \\
SLC3A2 & chr11 & $62,881,290$ & $1.31 \times 10^{-5}$ & $2.16 \times 10^{-2}$ & 0.256 \\
ARIH2 & chr3 & $48,928,674$ & $1.37 \times 10^{-5}$ & $1.18 \times 10^{-2}$ & 0.071 \\
DVL3 & chr3 & $184,164,517$ & $1.50 \times 10^{-5}$ & $2.16 \times 10^{-2}$ & -0.269 \\
DEF8 & chr16 & $89,959,366$ & $1.62 \times 10^{-5}$ & $1.18 \times 10^{-2}$ & -0.232 \\
SIAH2 & chr3 & $150,742,487$ & $1.62 \times 10^{-5}$ & $2.16 \times 10^{-2}$ & -0.362 \\
SNHG7 & chr9 & $136,724,659$ & $1.84 \times 10^{-5}$ & $2.16 \times 10^{-2}$ & 0.020 \\
LTBP2 & chr14 & $74,506,705$ & $1.94 \times 10^{-5}$ & $2.16 \times 10^{-2}$ & -0.248 \\
S100A16 & chr1 & $153,606,974$ & $2.29 \times 10^{-5}$ & $2.35 \times 10^{-2}$ & 0.200 \\
\hline
\end{tabular}

\subsection{Differential Gene/Transcript Expression}

In differential expression analysis, 991 genes and 1443 transcripts show higher expression in mock-treated cells, whereas 717 genes and 933 transcripts show higher expression in SARS-CoV-2 infected cells. These differentially expressed genes and transcripts can be served as molecular signatures for COVID-19 outcome prediction. The top 100 differentially expressed transcripts and genes were selected for bi-clustering analysis. The z-score transformation was applied to the expression value for each entry in the heatmap. From the results, we can see that the six samples are precisely clustered into two groups (Figures 6 and $\mathrm{S} 1$ in the Supplementary Materials). 

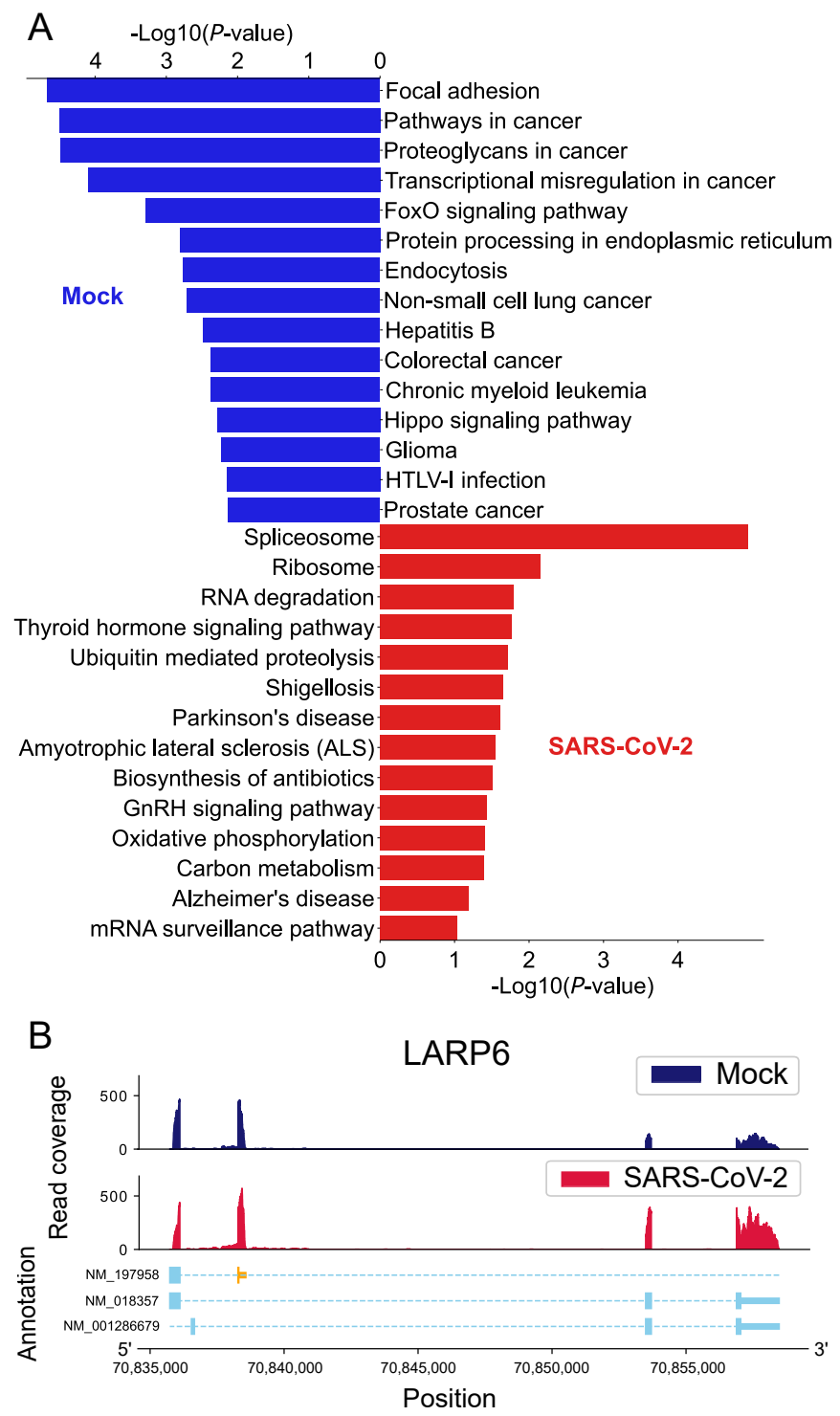

Figure 5. (A) KEGG pathways enriched by CR-APA events. The blue and red bar charts show the pathways enriched by the APA events in mock-treated cells and SARS-CoV-2 infected cells, respectively. (B) One example of a gene ( $L A R P 6)$ shows an alternative polyadenylation event in the coding region. The truncated coding exon is highlighted in orange.

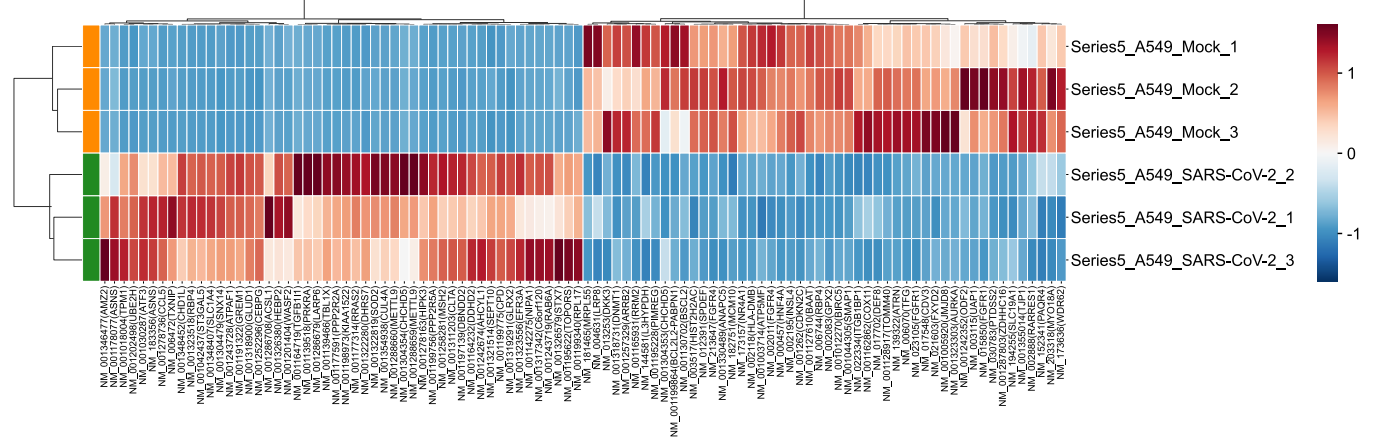

Figure 6. COVID-19 A549 cell lines are clustered by the top 100 transcript markers detected by differential transcript expression analysis.

Table 3 shows two differentially expressed genes that are related to SARS-CoV-2 pathogenesis and prognosis. Figures 7 and S2 in the Supplementary Materials show the 
enriched KEGG pathways by the differentially expressed transcripts and genes, respectively. In each figure, the top bar plot represents the pathways enriched by the transcripts or genes that are highly expressed in mock-treated cells, and the bottom bar plot represents the transcripts or genes that are highly expressed in SARS-CoV-2 infected cells. The enriched pathways by the gene expression and transcript expression analyses are similar. The KEGG pathway enrichment of the differentially expressed transcripts in the SARS-CoV-2 in Figure 7 shows an up-regulation in the pro-inflammatory pathways TNF- $\alpha$ and NF-kappa, confirming the finding that patients with SARS-CoV-2 were reported to have elevated levels of cytokines in blood and cytokine gene expression in peripheral blood mononuclear cells (PBMC) [32-34].

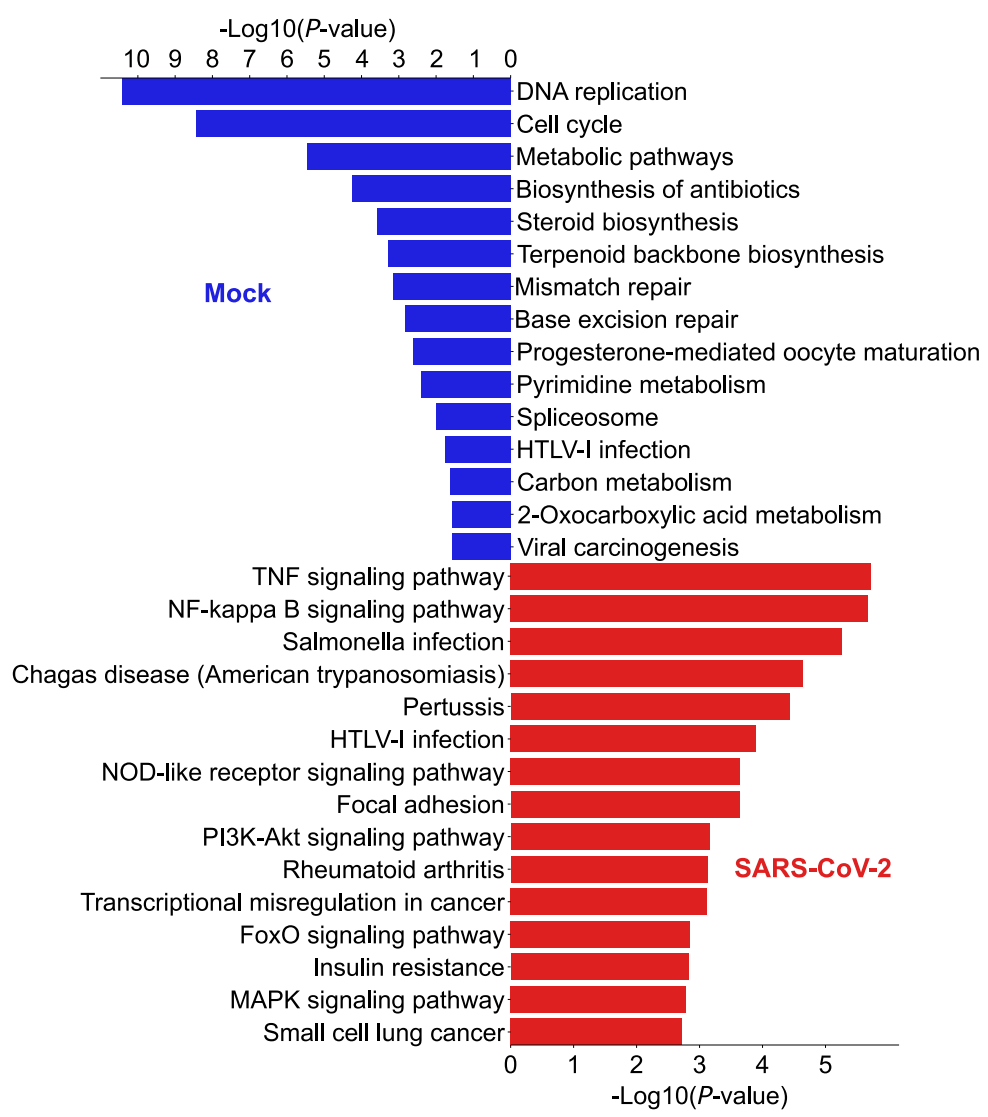

Figure 7. Differentially expressed transcripts enriched KEGG pathways. The blue and red bar charts show the pathways enriched by the up-regulated and down-regulated transcripts in mock-treated samples over SARS-CoV-2 infected samples, respectively.

\subsection{Comparison between Alternative Processing of Pre-mRNA and Differential Gene Expression}

In addition to mRNA expression, post-transcriptional regulation of gene expression (i.e., AS and APA) plays a critical role in the control of cellular processes and affects phenotypic changes in biological features [35]. They may serve as a new layer of biomarker for drug development for COVID-19 therapeutics. Therefore, it is important to compare the genes that are differentially expressed and the genes that undergo alternative processing events.

The relationship between differential gene expression and the UTR-APA event was tested based on the APA events identified in Section 2.3.1 and the differentially expressed genes identified in Section 2.4. We combined the analyses of UTR-APA and differential gene expression, and the results are shown in Figure 8A. Each gene was plotted by fold changes in the differential gene expression ( $y$-axis) and the significance of UTR-APA events ( $x$-axis). The left three sections and the right three sections in Figure 8A show the $3^{\prime}$-UTR-truncated genes in mock-treated cells and SARS-CoV-2 infected cells, respectively. The top three 
sections and the bottom three sections represent the up-regulated and down-regulated genes in SARS-CoV-2 infected cells over mock treated cells, respectively. From the plot, we can see that a significant portion $(>88 \%)$ of the genes showing UTR-APA events are not differentially expressed, indicating no strong correlation between the differential gene expression and the UTR-APA events in the SARS-CoV-2 infected cells. Similarly, more than $90 \%$ of the genes showing CR-APA events are not differentially expressed (Figure $8 \mathrm{~B}$ ). Therefore, the profiling of APA-based molecular signatures could provide additional predictive power and potentially lead to the findings of new target pathways for SARS-CoV-2 related translational research.

A

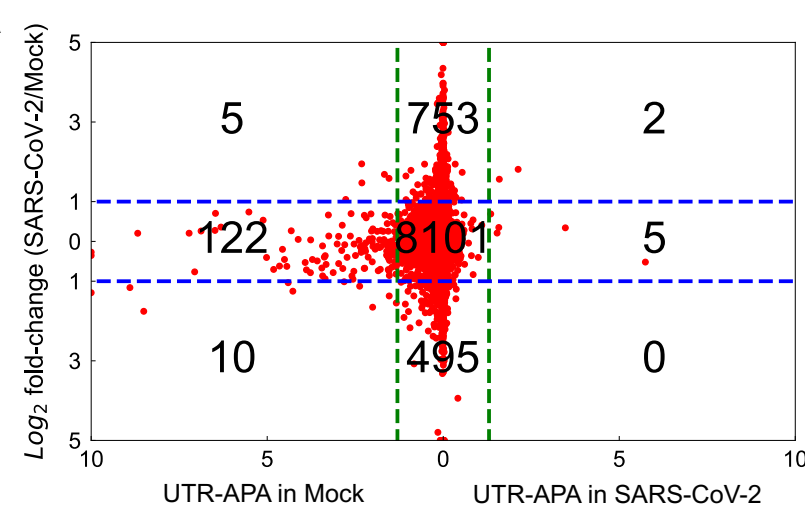

B

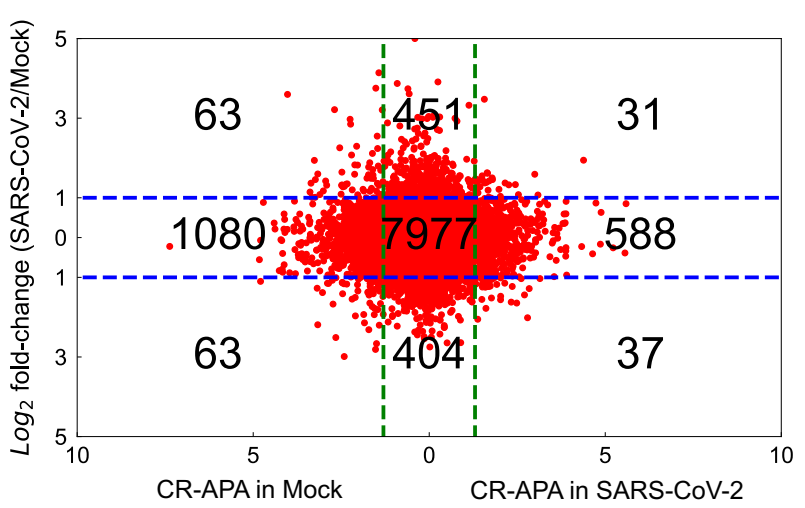

Figure 8. Scatter plot of APA and differential gene expression. Red dots represent the individual gene in the analysis. Horizontal blue-dashed lines represent the cutoff values for two-fold changes in differential gene expression. Vertical green-dashed lines represent the cutoff values for the $\log _{10}\left(p\right.$-value) of UTR-APA (A) determined by the Chi-squared test, and the $\log _{10}(p$-value) of CR-APA (B) determined by the Student's $t$-test.

To further investigate the relationship among all transcript variants identified in this study, a four-set Venn diagram is generated to illustrate the intersections of differentially expressed genes (DEG) and the genes with post-transcriptional regulation events (Figure 9). Both RefSeq and UCSC annotations were applied in the data analysis to make the experimental results comparable. The total number of events generated from alternative splicing, UTR-APA, CR-APA, and differentially expressed genes are 320,144, 1862, and 2256, respectively. None of the genes were shown in all four categories. Though 2256 genes were detected as differentially expressed genes, only 25 of them have alternative splicing events and 17 of them have UTR-APA events. In the case of an alternative splicing event in a gene showing CR-APA, it has a higher chance of being considered as a CR-APA gene. Based on these observations, we found that $27.5 \%$ of genes with AS events show CR-APA. Overall, the results further confirm that post-transcriptional regulation profiles and differential gene expressions profiles provide distinctive molecular signatures for COVID-19 
studies. In addition, the three different types of post-transcriptional regulation events barely overlap.

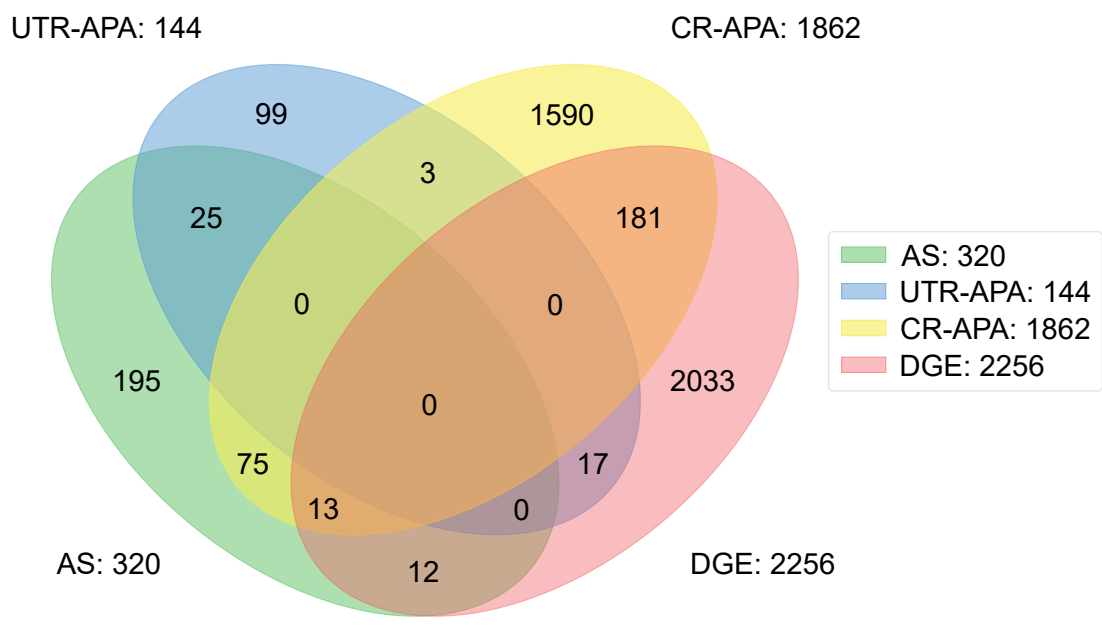

Figure 9. Four-set Venn diagram shows the overlapped genes in three different types of posttranscriptional regulations and differentially expressed genes (DEG).

\section{Discussion and Conclusions}

At present, the whole world is suffering from the COVID-19 pandemic, but the molecular mechanisms of COVID-19 pathogenesis are not clear. To better understand the molecular basis of the disease, here we compare the effects of transcriptional and post-transcriptional events on SARS-CoV-2 infection in lung cancer cell lines. From these analyses, we were able to identify distinct molecular signatures that were not otherwise found by traditional differential gene expression analysis. These novel molecular signatures may contribute to COVID-19 pathobiology. The results from the data analyses demonstrate that several transcriptional and post-transcriptional signatures identified in this study are highly correlated with SARS-CoV-2 pathogenesis and prognosis. These signatures are enriched in diverse biological pathways that were not detected in the original study [1], which only focused on differential gene expression analysis. These pathways may lead to the discovery of new molecular mechanisms of SARS-CoV-2 pathogenesis. In addition, post-transcriptional gene regulations provide additional molecular signatures for COVID19 therapeutic targets compared to the transcriptional signatures of COVID-19 patients. The latest COVID-19 study [10] has shown that the SARS-CoV-2 NSP16 protein suppresses mRNA splicing, leading to intron retention in host genes. In our data analyses, we only identified 16 intron retention events in the SARS-CoV-2 infected samples. This might be due to the fact that our custom-developed AS-Quant pipeline detects intron retention based on the annotations [26]. Thus, more cases for intron retention could be discovered by future pipelines, which will be important to identify non-canonical peptides produced from the translation of intron retained transcripts.

While our findings are confined at the transcriptome level, further investigations on the changes of proteome and post-translational modifications in the proteome are warranted to comprehensively dissect the mechanistic aspect of COVID-19 pathobiology. In our pathway enrichment analysis, genes in the ribosome pathway are up-regulated in the SARS-CoV-2 infected cells, suggesting that there may be an up-regulation of protein synthesis. Recent quantitative proteomics studies have suggested that time-dependent proteome changes in the post-infection of SARS-Cov-2 [36,37]. Studies have reported that in an early stage of infection (less than $24 \mathrm{~h}$ ), a proteome change occurs bi-directionally (some up-regulated and some down-regulated). However, at a later stage of infection (greater than $24 \mathrm{~h}$ ), there is an overall down-regulation of the global protein abundance. However, proteins that displayed differential phosphorylation and ubiquitination did not change the overall protein abundance. Nonetheless, $17.0 \%, 20.1 \%$, and $16.4 \%$ of the 
genes that contain AS, UTR-APA, and CR-APA events are reported to be differentially expressed in their protein levels [37]. In addition, both our post-transcriptional study and the proteomics studies have consistently identified the activation of the MAPK signaling pathway in SARS-CoV-2 infected cells. These findings suggest the importance of using a multi-omics approach in resolving detailed molecular and cellular signatures of COVID-19.

\section{Materials and Methods}

In this section, we first introduce the pipelines and strategies to detect the alternative splicing and alternative polyadenylation events between two biological conditions in Sections 4.1 and 4.2, respectively. Then, we explain the method to determine the differentially expressed transcripts and genes in Section 4.3. Finally, we provide an overview of the gene set enrichment analysis on the significant events in Section 4.4.

\subsection{Detection of Alternative Splicing Events}

To analyze the alternative splicing events between mock-treated and infected with SARS-CoV-2 samples with RNA-seq data, we used the computational pipeline AS-Quant [26]. Based on the read coverage files generated by SAMtools, all the potential splicing events were categorized into five major types of alternative splicing, as shown in Figure 1A. For each spliced exon, the average read coverage $(n)$ is calculated by the read count on that exon $\left(r_{e}\right)$ divided by the read length $\left(l_{e}\right)$ in AS-Quant:

$$
n=\frac{r_{e}}{l_{e}}
$$

The average read coverage for all other exons of that gene $(N)$ is calculated in the same way, by dividing the total reads $\left(r_{g}\right)$ by the gene length $\left(l_{g}\right)$. Next, the ratio difference between two biological conditions is calculated based on the following equation:

$$
\frac{n_{1}}{N_{1}}-\frac{n_{2}}{N_{2}},
$$

where 1 and 2 represent the different conditions. The most significant events are reported according to the $p$-value generated by a canonical $2 \times 2$ Chi-squared test. The topmost events are then illustrated by read coverage plots to give a visual representation (e.g., Figure 2B).

\subsection{Detection of APA Events}

\subsubsection{UTR-APA}

To identify the 3'-UTR-APA events between mock-treated or SARS-CoV-2 infected samples with RNA-seq data, we applied the computational tool APA-Scan [27]. APA-Scan utilizes either predicted or experimentally validated polyadenylation signals as a reference for 3'-UTR polyadenylation sites. It also calculates the amount of full-length and truncated $3^{\prime}$-UTR transcripts with the RNA-seq data.

The canonical polyadenylation signals (PAS) AATAAA and ATTAAA in the distal $3^{\prime}$-UTR region were considered as the tentative locations of cleavage sites. Then, the APAevents were evaluated by comparing the RNA-seq reads coverage upstream and downstream of the candidate cleavage site between the samples in two different biological conditions. Specifically, the average read coverage for upstream $\left(M_{1}\right.$ and $M_{2}$ for two biological conditions) and downstream $\left(m_{1}\right.$ and $\left.m_{2}\right)$ of the candidate cleavage site were calculated. Next, the ratio difference between two conditions was determined by the following equation:

$$
\frac{m_{1}}{M_{1}}-\frac{m_{2}}{M_{2}} .
$$

The $2 \times 2$ Chi-square test was then applied to assess the significance of the candidate events. The read coverage plots of the significant events were reported according to the user-defined $p$-value cutoff (e.g., Figure 3). 


\subsubsection{CR-APA}

To identify and quantify dynamic CR-APA events, the protein-coding transcripts of a gene were categorized as either full-length or truncated transcripts based on the NCBI human hg38 RefSeq [28] or UCSC annotations [38]. A transcript is categorized as a truncated transcript if it satisfies the following conditions: (1) its coding end is not the maximum coding end in the gene, and (2) its transcript end is not the maximum transcript end in the gene; otherwise, the transcript is a full-length transcript in the gene. The CR-truncation ratio $R_{C R}$ that quantifies the CR-APA event is defined as,

$$
R_{C R}=E_{C R} / E_{A L L}
$$

where $E_{C R}$ is the summation of expressions of all truncated transcripts in the gene, $E_{A L L}$ denotes gene expression, and $R_{C R} \in[0,1]$. The Student's $t$-test is then applied to the $\mathrm{CR}$-truncation ratios to determine the significant $\mathrm{CR}$-APA events between the two groups of samples.

\subsection{Differential Gene/Transcript Expressions}

Differential gene expression analysis has been widely used to identify transcriptomic signatures that define phenotypic changes in biology. In this work, Kallisto [12] was applied to perform the quantification of RNA-seq data for COVID-19 samples. After filtering out lowly expressed genes $(\mathrm{TPM}<1)$, the $\log _{2}$ fold change of transcript or gene expression was calculated between two groups of samples. The transcripts or genes with a $\log _{2}$ fold change larger than 1 or smaller than -1 , and with a Student's $t$-test $p$-value $<0.05$ were considered as significantly differentially expressed.

\subsection{Enriched Pathway Analysis}

After identifying the AS events, APA events, and differentially expressed genes/transcripts in each biological condition, the events and genes which are overrepresented in GO (Gene Ontology) terms and KEGG (Kyoto Encyclopedia of Genes and Genomes) pathways were determined by DAVID Bioinformatics Resources [39]. These enriched functional profiles and molecular interactions could help us better understand the underlying biological processes in COVID-19. The KEGG pathway is a collection of pathway-maps representing molecular level information, including metabolism, genetic information processing, environmental information processing, cellular processes, organismal systems, human diseases, and drug development. The Gene Ontology (GO) terms define the biological domain with respect to three aspects: molecular function, cellular component, and biological process.

Supplementary Materials: The following are available at https://www.mdpi.com/article/10.3390/ ijms22189684/s1.

Author Contributions: J.S., N.A.F., D.F., J.Y. and W.Z. conceived the study and planned the analysis. J.S. and N.F. performed data analysis. J.S., N.A.F., H.N., S.C., I.M., J.Y. and W.Z. wrote the manuscript. All authors have read and agreed to the published version of the manuscript.

Funding: The study was supported by the National Science Foundation grant IIS1755761 and FET2003749 and National Institutes of Health 2R01GM113952. Publication costs are funded by the National Science Foundation grant FET2003749.

Institutional Review Board Statement: Not applicable.

Informed Consent Statement: Not applicable.

Data Availability Statement: The dataset GSE147507 used in this study is available at the NCBI GEO Database (accessed on 15 April 2020).

Conflicts of Interest: The authors declare no conflict of interest. 


$\begin{array}{ll}\text { Abbreviations } \\ \text { RT-PCR } & \text { Real-Time Polymerase Chain Reaction } \\ \text { CR-APA } & \text { Coding Region Alternative PolyAdenylation } \\ \text { UTR-APA } & \text { UnTranslated Region Alternative PolyAdenylation } \\ \text { AS-Quant } & \text { Alternative Splicing Quantitation } \\ \text { APA-Scan } & \text { Alternative Polyadenylation Scan } \\ \text { PAS } & \text { PolyAdenylation Signal } \\ \text { DEG } & \text { Differentially Expressed Genes } \\ \text { MAPK } & \text { Mitogen-Activated Protein Kinase } \\ \text { GEO } & \text { Gene Expression Omnibus } \\ \text { GO } & \text { Gene Ontology } \\ \text { KEGG } & \text { Kyoto Encyclopedia of Genes and Genomes }\end{array}$

\section{References}

1. Blanco-Melo, D.; Nilsson-Payant, B.E.; Liu, W.C.; Uhl, S.; Hoagland, D.; Møller, R.; Jordan, T.X.; Oishi, K.; Panis, M.; Sachs, D.; et al. Imbalanced host response to SARS-CoV-2 drives development of COVID-19. Cell 2020, 181, 1036-1045.e9. [CrossRef]

2. Sun, J.; Ye, F.; Wu, A.; Yang, R.; Pan, M.; Sheng, J.; Zhu, W.; Mao, L.; Wang, M.; Xia, Z.; et al. Comparative transcriptome analysis reveals the intensive early stage responses of host cells to SARS-CoV-2 infection. Front. Microbiol. 2020, 11, 2881. [CrossRef]

3. Moni, M.A.; Quinn, J.M.; Sinmaz, N.; Summers, M.A. Gene expression profiling of SARS-CoV-2 infections reveal distinct primary lung cell and systemic immune infection responses that identify pathways relevant in COVID-19 disease. Brief. Bioinform. 2021, 22, 1324-1337. [CrossRef]

4. Lieberman, N.A.; Peddu, V.; Xie, H.; Shrestha, L.; Huang, M.L.; Mears, M.C.; Cajimat, M.N.; Bente, D.A.; Shi, P.Y.; Bovier, F.; et al. In vivo antiviral host transcriptional response to SARS-CoV-2 by viral load, sex, and age. PLoS Biol. 2020, 18, e3000849. [CrossRef]

5. Thompson, M.G.; Dittmar, M.; Mallory, M.J.; Bhat, P.; Ferretti, M.B.; Fontoura, B.M.; Cherry, S.; Lynch, K.W. Viral-induced alternative splicing of host genes promotes influenza replication. eLife 2020, 9, e55500. [CrossRef]

6. Boudreault, S.; Roy, P.; Lemay, G.; Bisaillon, M. Viral modulation of cellular RNA alternative splicing: A new key player in virus-host interactions? Wiley Interdiscip. Rev. RNA 2019, 10, e1543. [CrossRef]

7. Su, Z.; Huang, D. Alternative Splicing of Pre-mRNA in the Control of Immune Activity. Genes 2021, 12, 574. [CrossRef]

8. Tsai, P.L.; Chiou, N.T.; Kuss, S.; García-Sastre, A.; Lynch, K.W.; Fontoura, B.M. Cellular RNA binding proteins NS1-BP and hnRNP K regulate influenza A virus RNA splicing. PLoS Pathog. 2013, 9, e1003460. [CrossRef] [PubMed]

9. Kalam, H.; Singh, K.; Chauhan, K.; Fontana, M.F.; Kumar, D. Alternate splicing of transcripts upon Mycobacterium tuberculosis infection impacts the expression of functional protein domains. IUBMB Life 2018, 70, 845-854. [CrossRef] [PubMed]

10. Banerjee, A.K.; Blanco, M.R.; Bruce, E.A.; Honson, D.D.; Chen, L.M.; Chow, A.; Bhat, P.; Ollikainen, N.; Quinodoz, S.A.; Loney, C.; et al. SARS-CoV-2 disrupts splicing, translation, and protein trafficking to suppress host defenses. Cell 2020, 183, 1325-1339. [CrossRef] [PubMed]

11. Kim, D.; Pertea, G.; Trapnell, C.; Pimentel, H.; Kelley, R.; Salzberg, S.L. TopHat2: Accurate alignment of transcriptomes in the presence of insertions, deletions and gene fusions. Genome Biol. 2013, 14, R36. [CrossRef]

12. Bray, N.L.; Pimentel, H.; Melsted, P.; Pachter, L. Near-optimal probabilistic RNA-seq quantification. Nat. Biotechnol. 2016, 34, 525-527. [CrossRef]

13. Li, H.; Handsaker, B.; Wysoker, A.; Fennell, T.; Ruan, J.; Homer, N.; Marth, G.; Abecasis, G.; Durbin, R. The sequence alignment/map format and SAMtools. Bioinformatics 2009, 25, 2078-2079. [CrossRef] [PubMed]

14. Huang, D.W.; Sherman, B.T.; Lempicki, R.A. Bioinformatics enrichment tools: Paths toward the comprehensive functional analysis of large gene lists. Nucleic Acids Res. 2009, 37, 1-13. [CrossRef]

15. Li, Q.; Wang, L.; Dong, C.; Che, Y.; Jiang, L.; Liu, L.; Zhao, H.; Liao, Y.; Sheng, Y.; Dong, S.; et al. The interaction of the SARS coronavirus non-structural protein 10 with the cellular oxido-reductase system causes an extensive cytopathic effect. J. Clin. Virol. 2005, 34, 133-139. [CrossRef]

16. Carbajo-Lozoya, J.; Müller, M.A.; Kallies, S.; Thiel, V.; Drosten, C.; Von Brunn, A. Replication of human coronaviruses SARS-CoV, HCoV-NL63 and HCoV-229E is inhibited by the drug FK506. Virus Res. 2012, 165, 112-117. [CrossRef]

17. Wang, J.; Shi, C.; Xu, Q.; Yin, H. SARS-CoV-2 nucleocapsid protein undergoes liquid-liquid phase separation into stress granules through its N-terminal intrinsically disordered region. Cell Discov. 2021, 7, 1-5. [CrossRef]

18. Fang, Y.T.; Lin, C.F.; Liao, P.C.; Kuo, Y.M.; Wang, S.; Yeh, T.M.; Shieh, C.C.K.; Su, I.J.; Lei, H.Y.; Lin, Y.S. Annexin A2 on lung epithelial cell surface is recognized by severe acute respiratory syndrome-associated coronavirus spike domain 2 antibodies. Mol. Immunol. 2010, 47, 1000-1009. [CrossRef]

19. Szczepanski, A.; Owczarek, K.; Milewska, A.; Baster, Z.; Rajfur, Z.; Mitchell, J.A.; Pyrc, K. Canine respiratory coronavirus employs caveolin-1-mediated pathway for internalization to HRT-18G cells. Vet. Res. 2018, 49, 1-14. [CrossRef]

20. Riad, A.; Aubert, Y.; Zeng, C.; Graham, T.J.; Petersson, E.J.; Capell, B.C.; Mach, R.H. Differential gene expression by RNA-Seq in Sigma-2 Receptor/TMEM97 knockout cells reveals its role in complement activation and SARS-CoV-2 viral uptake. bioRxiv 2021. [CrossRef] 
21. Korkmaz, B.; Lesner, A.; Marchand-Adam, S.; Moss, C.; Jenne, D.E. Lung Protection by Cathepsin C Inhibition: A New Hope for COVID-19 and ARDS? Miniperspective. J. Med. Chem. 2020, 63, 13258-13265. [CrossRef]

22. Abedi, F.; Rezaee, R.; Karimi, G. Plausibility of therapeutic effects of Rho kinase inhibitors against Severe Acute Respiratory Syndrome Coronavirus 2 (COVID-19). Pharmacol. Res. 2020, 156, 104808. [CrossRef] [PubMed]

23. Fukushi, M.; Yoshinaka, Y.; Matsuoka, Y.; Hatakeyama, S.; Ishizaka, Y.; Kirikae, T.; Sasazuki, T.; Miyoshi-Akiyama, T. Monitoring of $\mathrm{S}$ protein maturation in the endoplasmic reticulum by calnexin is important for the infectivity of severe acute respiratory syndrome coronavirus. J. Virol. 2012, 86, 11745-11753. [CrossRef]

24. Inde, Z.; Yapp, C.; Joshi, G.N.; Spetz, J.; Fraser, C.; Deskin, B.; Ghelfi, E.; Sodhi, C.; Hackam, D.J.; Kobzik, L.; et al. Age-dependent regulation of SARS-CoV-2 cell entry genes and cell death programs correlates with COVID-19 disease severity. bioRxiv 2020. [CrossRef]

25. Gassen, N.C.; Niemeyer, D.; Muth, D.; Corman, V.M.; Martinelli, S.; Gassen, A.; Hafner, K.; Papies, J.; Mösbauer, K.; Zellner, A.; et al. SKP2 attenuates autophagy through Beclin1-ubiquitination and its inhibition reduces MERS-Coronavirus infection. Nat. Commun. 2019, 10,1-16.

26. Fahmi, N.A.; Nassereddeen, H.; Chang, J.; Park, M.; Yeh, H.; Sun, J.; Fan, D.; Yong, J.; Zhang, W. As-quant: Detection and visualization of alternative splicing events with rna-seq data. Int. J. Mol. Sci. 2021, 22, 4468. [CrossRef]

27. Fahmi, N.A.; Chang, J.W.; Nassereddeen, H.; Ahmed, K.T.; Fan, D.; Yong, J.; Zhang, W. APA-Scan: Detection and Visualization of 3'-UTR APA with RNA-seq and $3^{\prime}$-end-seq Data. bioRxiv 2020. [CrossRef]

28. O'Leary, N.A.; Wright, M.W.; Brister, J.R.; Ciufo, S.; Haddad, D.; McVeigh, R.; Rajput, B.; Robbertse, B.; Smith-White, B.; Ako-Adjei, D.; et al. Reference sequence (RefSeq) database at NCBI: Current status, taxonomic expansion, and functional annotation. Nucleic Acids Res. 2016, 44, D733-D745. [CrossRef]

29. Rosenbloom, K.R.; Sloan, C.A.; Malladi, V.S.; Dreszer, T.R.; Learned, K.; Kirkup, V.M.; Wong, M.C.; Maddren, M.; Fang, R.; Heitner, S.G.; et al. ENCODE data in the UCSC Genome Browser: Year 5 update. Nucleic Acids Res. 2012, 41, D56-D63. [CrossRef] [PubMed]

30. Cloonan, S.M.; Choi, A.M. Mitochondria in lung disease. J. Clin. Investig. 2016, 126, 809-820. [CrossRef]

31. Shiozawa, Y.; Malcovati, L.; Gallì, A.; Sato-Otsubo, A.; Kataoka, K.; Sato, Y.; Watatani, Y.; Suzuki, H.; Yoshizato, T.; Yoshida, K.; et al. Aberrant splicing and defective mRNA production induced by somatic spliceosome mutations in myelodysplasia. Nat. Commun. 2018, 9, 1-16. [CrossRef]

32. Sheng, W.H.; Chiang, B.L.; Chang, S.C.; Ho, H.N.; Wang, J.T.; Chen, Y.C.; Hsiao, C.H.; Hseuh, P.R.; Chie, W.C.; Yang, P.C. Clinical manifestations and inflammatory cytokine responses in patients with severe acute respiratory syndrome. J. Formos. Med Assoc. 2005, 104, 715-723. [PubMed]

33. Yu, S.Y.; Hu, Y.W.; Liu, X.Y.; Xiong, W.; Zhou, Z.T.; Yuan, Z.H. Gene expression profiles in peripheral blood mononuclear cells of SARS patients. World J. Gastroenterol. WJG 2005, 11, 5037. [CrossRef] [PubMed]

34. Zhang, Y.; Li, J.; Zhan, Y.; Wu, L.; Yu, X.; Zhang, W.; Ye, L.; Xu, S.; Sun, R.; Wang, Y.; et al. Analysis of serum cytokines in patients with severe acute respiratory syndrome. Infect. Immun. 2004, 72, 4410-4415. [CrossRef] [PubMed]

35. Ahmed, K.; Sun, J.; Chen, W.; Martinez, I.; Cheng, S.; Zhang, W.; Yong, J. In silico model for miRNA-mediated regulatory network in cancer. Brief. Bioinform. 2021. [CrossRef] [PubMed]

36. Bouhaddou, M.; Memon, D.; Meyer, B.; White, K.M.; Rezelj, V.V.; Marrero, M.C.; Polacco, B.J.; Melnyk, J.E.; Ulferts, S.; Kaake, R.M.; et al. The global phosphorylation landscape of SARS-CoV-2 infection. Cell 2020, 182, 685-712. [CrossRef] [PubMed]

37. Stukalov, A.; Girault, V.; Grass, V.; Karayel, O.; Bergant, V.; Urban, C.; Haas, D.A.; Huang, Y.; Oubraham, L.; Wang, A.; et al. Multilevel proteomics reveals host perturbations by SARS-CoV-2 and SARS-CoV. Nature 2021, 594, 246-252. [CrossRef] [PubMed]

38. Fujita, P.A.; Rhead, B.; Zweig, A.S.; Hinrichs, A.S.; Karolchik, D.; Cline, M.S.; Goldman, M.; Barber, G.P.; Clawson, H.; Coelho, A.; et al. The UCSC genome browser database: Update 2011. Nucleic Acids Res. 2010, 39, D876-D882. [CrossRef]

39. Huang, D.W.; Sherman, B.T.; Lempicki, R.A. Systematic and integrative analysis of large gene lists using DAVID bioinformatics resources. Nat. Protoc. 2009, 4, 44. [CrossRef] 